\title{
COULD THE CLASSICAL RELATIVISTIC ELECTRON BE A STRANGE ATTRACTOR?
}

\author{
L. P. HORWITZ, N. KATZ, AND O. ORON
}

Received 5 January 2004

We review the formulation of the problem of the electromagnetic self-interaction of a relativistic charged particle in the framework of the manifestly covariant classical mechanics of Stueckelberg, Horwitz, and Piron. The gauge fields of this theory, in general, cause the mass of the particle to change. We show that the nonlinear Lorentz force equation for the self-interaction resulting from the expansion of Green's function has chaotic solutions. We study the autonomous equation for the off-shell particle mass here, for which the effective charged particle mass achieves a macroscopic average value determined by what appears to be a strange attractor.

\section{Introduction}

The advent of powerful computers in the second half of the twentieth century has made it attractively possible to investigate physical problems that involve a high level of intrinsic nonlinearity. The remarkable discovery by Lorenz in 1963 [12] that rather simple nonlinear systems are capable of displaying highly complex, unstable, and, in many cases, beautiful systems of orbits gave rise to the subject of chaos, now under intensive study both in specific applications and in its general properties. The instability of these orbits is characterized by the fact that small variations of initial conditions generate new orbits that diverge from the original orbit exponentially.

Such studies range from nonlinear dynamical systems, both dissipative and nondissipative, involving deterministic chaos, and hard surface-type collisions (e.g., billiards), to the study of fluctuation phenomena in quantum field theory [3]. The results have led to a deeper understanding of turbulence in hydrodynamics, the behavior of nonlinear electrical circuits, plasmas, biological systems, and chemical reactions. It has, furthermore, given deep insights into the foundations of statistical mechanics.

What appears to be a truly striking fact that has emerged from this experience is that the appearance of what has become to be known as chaotic behavior is not just the property of some special systems designed for the purpose of achieving some result depending on the nonlinearity, such as components of an electric circuit, but seems to occur almost 
universally in our perception of the physical world. The potentialities presented by this subject are therefore very extensive and provide a domain for discovery that appears to be virtually unlimited.

As an example of the occurrence of chaotic behavior in one of the most fundamental and elementary systems in nature, we wish to discuss here the results of our study of the classical relativistic charged particle (e.g., the classical electron without taking into account the dynamical effects of its spin, an intrinsically quantum effect).

There remains, in the area of research on chaotic systems, the question of making a correspondence between classical chaotic behavior and the properties of the corresponding quantum systems. Much is known on the signatures of chaos for quantum systems, for example, the occurrence of Wigner distributions in the spectra for Hamiltonian chaos. The study of relativistic phenomena, of the type we will consider here, where the explicit self-interaction problem gives rise to instability, may have a counterpart in the renormalization program in quantum field theory. The existence of radiation due to the accelerated motion of the particle raises the question of how this radiation, acting back on the particle, affects its motion. Rohrlich [19] has described the historical development of this problem, where the first steps were taken by Abraham in 1905 [1], culminating in the work of Dirac [5], who derived the equation for the ideal point electron in the form

$$
m \frac{d^{2} x^{\mu}}{d s^{2}}=F_{\nu}^{\mu} \frac{d x^{\mu}}{d s}+\Gamma^{\mu}
$$

where $m$ is the electron mass, including electromagnetic correction, $s$ is the proper time along the trajectory $x^{\mu}(s)$ in spacetime, $F_{\nu}^{\mu}$ is the covariant form of the electromagnetic force tensor, $e$ is the electron charge, and

$$
\Gamma^{\mu}=\frac{2}{3} \frac{e^{2}}{c^{3}}\left(\frac{d^{3} x^{\mu}}{d s^{3}}-\frac{d^{2} x^{\nu}}{d s^{2}} \frac{d^{2} x_{v}}{d s^{2}} \frac{d x^{\mu}}{d s}\right)
$$

Here, the indices $\mu, \nu$, running over $0,1,2,3$, label the spacetime variables that represent the action of the Lorentz group; the index raising and lowering Lorentz invariant tensor $\eta_{\mu \nu}$ is of the form $\operatorname{diag}(-1,+1,+1,+1)$. The expression for $\Gamma^{\mu}$ was originally found by Abraham in 1905 [1], shortly after the discovery of special relativity, and is known as the Abraham four-vector of radiation reaction. Dirac's derivation [5] was based on a direct application of Green's functions for the Maxwell fields, obtaining the form (1.1), which we will call the Abraham-Lorentz-Dirac equation. In this calculation, Dirac used the difference between retarded and advanced Green's functions so as to eliminate the singularity carried by each. Sokolov and Ternov [25], for example, give a derivation using the retarded Green's function alone, and show how the singular term can be absorbed into the mass $m$.

Formula (1.1) contains a so-called singular perturbation problem. There is a small coefficient multiplying a derivative of higher order than that of the unperturbed problem; since the highest-order derivative is the most important in the equation, dividing by $e^{2}$, one sees that any small deviation in the lower-order terms results in a large effect on the 
orbit, and there are unstable solutions, often called "runaway solutions." There is a large literature (see $[2,29]$ and the references therein) on the methods of treating this instability, and there is considerable discussion in Rohrlich's book [19] as well (he describes a method for eliminating the unstable solutions by studying asymptotic properties).

The existence of these runaway solutions, for which the electron undergoes an exponential acceleration with no external force beyond a short initial perturbation of the free motion, is a difficulty for the point electron picture in the framework of the Maxwell theory with the covariant Lorentz force. Rohrlich [20, 21, 22] (see also [11, 30]) has discussed the idea that the point electron idealization may not be really physical, based on arguments from classical and quantum theory, and emphasized that, for the corresponding classical problem, a finite size can eliminate this instability. Of course, this argument is valid, but it leaves open the question of the consistency of the Maxwell-Lorentz theory which admits the concept of point charges, as well as what is the nature of the elementary charges that make up the extended distribution.

It is quite remarkable that Gupta and Padmanabhan [7], using essentially geometrical arguments (solving the static problem in the frame of the accelerating particle with a curved background metric), have shown that the description of the motion of an accelerating charged particle must include the radiation terms of the Abraham-LorentzDirac equation. Recognizing that the electron's acceleration precludes the use of a sequence of "instantaneous" inertial frames to describe the action of the forces on the electron $[13,14,15]$, they carry out a Fermi-Walker transformation [16], going to an accelerating frame (assuming constant acceleration) in which the electron is actually inertial, and there solve the Coulomb problem in the curved coordinates provided by the Fermi-Walker transformation. Transforming back to laboratory coordinates, they find the Abraham-Lorentz-Dirac equation without the direct use of Maxwell Green's functions for the radiation field. This result, suggesting the relevance of curvature in the spacetime manifold such as that generated by sources in general relativity along with other more elementary manifestations of mass renormalization (such as the contribution to the mass due to electromagnetic interactions and the identification of Green's function singularity contribution with part of the electron mass), carries an implication that the electron mass may play an important dynamical role.

Stueckelberg, in $1941[27,28]$, proposed a manifestly covariant form of classical and quantum mechanics in which space and time become dynamical observables. They are therefore represented in quantum theory by operators on a Hilbert space on square integrable functions in space and time. The dynamical development of the state is controlled by an invariant parameter $\tau$, which one might call the world time, coinciding with the time on the (on-mass-shell) freely falling clocks of general relativity. Stueckelberg [27, 28] started his analysis by considering a classical world line, and argued that under the action of forces, the world line would not be straight, and in fact could be curved back in time. He identified the branch of the curve running backward in time with the antiparticle, a view taken also by Feynman in his perturbative formulation of quantum electrodynamics in 1948 [6]. Realizing that such a curve could not be parametrized by $t$ (for some values of $t$, there are two values of the space variables), Stueckelberg introduced the parameter $\tau$ along the trajectory. 
This parameter is not necessarily identical to proper time, even for inertial motion for which proper time is a meaningful concept. Stueckelberg postulated the existence of an invariant "Hamiltonian" $K$, which would generate Hamilton equations for the canonical variables $x^{\mu}$ and $p^{\mu}$ of the form

$$
\dot{x}^{\mu}=\frac{\partial K}{\partial p_{\mu}}, \quad \dot{p}^{\mu}=-\frac{\partial K}{\partial x_{\mu}},
$$

where the dot indicates differentiation with respect to $\tau$. Taking, for example, the model

$$
K_{0}=\frac{p^{\mu} p_{\mu}}{2 M}
$$

we see that the Hamilton equations imply that

$$
\dot{x}^{\mu}=\frac{p^{\mu}}{M}
$$

It then follows that

$$
\frac{d \mathbf{x}}{d t}=\frac{\mathbf{p}}{E}
$$

where $p^{0} \equiv E$, where we set the velocity of light $c=1$; this is the correct definition for the velocity of a free relativistic particle. It follows, moreover, that

$$
\dot{x}^{\mu} \dot{x}_{\mu}=\frac{d x^{\mu} d x_{\mu}}{d \tau^{2}}=\frac{p^{\mu} p_{\mu}}{M^{2}}
$$

With our choice of metric, $d x^{\mu} d x_{\mu}=-d s^{2}$ and $p^{\mu} p_{\mu}=-m^{2}$, where $m$ is the classical experimentally measured mass of the particle (at a given instant of $\tau$ ). We see from this that

$$
\frac{d s^{2}}{d \tau^{2}}=\frac{m^{2}}{M^{2}}
$$

and hence the proper time is not identical to the evolution parameter $\tau$. In the case that $m^{2}=M^{2}$, it follows that $d s=d \tau$, and we say that the particle is "on-shell."

For example, in the case of an external potential $V(x)$, where we write $x \equiv x^{\mu}$, the Hamiltonian becomes

$$
K=\frac{p^{\mu} p_{\mu}}{2 M}+V(x)
$$

so that, since $K$ is a constant of the motion, $m^{2}$ varies from point to point with the variations of $V(x)$. It is important to recognize from this discussion that the observable particle mass depends on the state of the system (in the quantum theory, the expectation value of the operator $p^{\mu} p_{\mu}$ provides the expected value of the mass squared). 
One may see, alternatively, that phenomenologically, the mass of a nucleon, such as the neutron, clearly depends on the state of the system. The free neutron is not stable but decays spontaneously into a proton, electron, and antineutrino since it is heavier than the proton. However, bound in a nucleus, it may be stable (in the nucleus, the proton may decay into neutron, positron, and neutrino, since the proton may be sufficiently heavier than the neutron). The mass of the bound electron (in interaction with the electromagnetic field), as computed in quantum electrodynamics, is different from that of the free electron, and the difference contributes to the Lamb shift. This implies that, if one wishes to construct a covariant quantum theory, the variables $E$ (energy) and $\mathbf{p}^{\mu}$ should be independent and not constrained by the relation $E^{2}=\mathbf{p}^{2}+m^{2}$, where $m$ is a fixed constant. This relation implies, moreover, that $m^{2}$ is a dynamical variable. It then follows, quantum mechanically through the Fourier relation between the energy-momentum representation of a wave function and the spacetime representation, that the variable $t$, along with the variable $\mathbf{x}$, is a dynamical variable. Classically, $t$ and $\mathbf{x}$ are recognized as variables of the phase space through the Hamilton equations.

Since, in nature, particles appear with fairly sharp mass values (not necessarily with zero spread), we may assume the existence of some mechanism which will drive the particle's mass back to its original mass-shell value (after the source responsible for the mass change ceases to act) so that the particle's mass shell is defined. We will not take such a mechanism into account explicitly here in developing the dynamical equations. We will assume that if this mechanism is working, it is a relatively smooth function (e.g., a minimum in free energy which is broad enough for our off-shell driving force to work fairly freely). (A relativistic Lee model has been worked out which describes a physical mass shell as a resonance, and therefore a stability point on the spectrum [8], but at this point, it is not clear to us how this mechanism works in general. It has been suggested by $\mathrm{T}$. Jordan [personal communication] that the definition of the physical mass shell could follow from the interaction of the particle with fields (a type of "self-interaction"); this mechanism could provide for perhaps more than one mass state for a particle, such as the electron and muon and the various types of neutrinos, but no detailed model has been so far studied.)

In an application of statistical mechanics to this theory [4], it has been found that a high temperature phase transition can be responsible for the restriction of the particle's mass (on the average, in equilibrium). In the present work, we will see that, at least in the classical theory, the nonlinear equations induced by radiation reaction may have a similar effect.

A theory of Stueckelberg type, providing a framework for dynamical evolution of a relativistic charged particle, therefore appears to be a natural dynamical generalization of the curved space formulation of Gupta and Padmanabhan [7] and the static picture of Dirac. We will study this in the following.

The Stueckelberg formulation implies the existence of a fifth "electromagnetic" potential through the requirement of gauge invariance, and there is a generalized Lorentz force which contains a term that drives the particle off-shell, whereas the terms corresponding to the electric and magnetic parts of the usual Maxwell fields do not (for the nonrelativistic case, the electric field may change the energy of a charged particle, but not the 
magnetic field; the electromagnetic field tensor in our case is analogous to the magnetic field, and the new field strengths, derived from the $\tau$ dependence of the fields and the additional gauge field, are analogous to the electric field, as we will see).

In the following, we give the structure of the field equations, and show that the standard Maxwell theory is properly contained in this more general framework. Applying Green's functions to the current source provided by the relativistic particle, and the generalized Lorentz force, we obtain equations of motion for the relativistic particle which is, in general, off-shell. As in Dirac's result, these equations are of third order in the evolution parameter, and therefore are highly unstable. However, the equations are very nonlinear, and give rise to chaotic behavior.

Our results exhibit what appears to be a strange attractor in the phase space of the autonomous equation for the off-mass-shell deviation. This attractor may stabilize the electron's mass in some neighborhood. We conjecture that it stabilizes the orbits macroscopically as well, but a detailed analysis awaits the application of more powerful computing facilities and procedures.

\section{Equations of motion}

The Stueckelberg-Schrödinger equation which governs the evolution of a quantum state over the manifold of spacetime was postulated by Stueckelberg $[27,28]$ to be, for the free particle,

$$
i \frac{\partial \psi_{\tau}}{\partial \tau}=\frac{p^{\mu} p_{\mu}}{2 M} \psi_{\tau}
$$

where, on functions of spacetime, $p_{\mu}$ is represented by $\partial / \partial x^{\mu} \equiv \partial_{\mu}$.

Taking into account full $U(1)$ gauge invariance corresponding to the requirement that the theory maintains its form under the replacement of $\psi$ by $e^{i e_{0} \Lambda} \psi$, the StueckelbergSchrödinger equation (including a compensation field for the $\tau$-derivative of $\Lambda$ ) is $[10,23,24]$

$$
\left(i \frac{\partial}{\partial \tau}+e_{0} a_{5}(x, \tau)\right) \psi_{\tau}(x)=\frac{\left(p^{\mu}-e_{0} a^{\mu}(x, \tau)\right)\left(p_{\mu}-e_{0} a_{\mu}(x, \tau)\right)}{2 M} \psi_{\tau}(x)
$$

where the gauge fields may depend on $\tau$, and $e_{0}$ is a coupling constant which we will see has the dimension $\ell^{-1}$. The corresponding classical Hamiltonian then has the form

$$
K=\frac{\left(p^{\mu}-e_{0} a^{\mu}(x, \tau)\right)\left(p_{\mu}-e_{0} a_{\mu}(x, \tau)\right)}{2 M}-e_{0} a_{5}(x, \tau)
$$

in place of (2.1). Stueckelberg [27, 28] did not take into account this full gauge invariance requirement, working in the analog of what is known in the nonrelativistic case as the Hamilton gauge (where the gauge function $\Lambda$ is restricted to be independent of time). The 
equations of motion for the field variables are given (for both the classical and quantum theories) by $[10,23,24]$

$$
\lambda \partial_{\alpha} f^{\beta \alpha}(x, \tau)=e_{0} j^{\beta}(x, \tau)
$$

where $\alpha, \beta=0,1,2,3,5$, the last corresponding to the $\tau$ index, and $\lambda$, of dimension $\ell^{-1}$, is a factor on the terms $f^{\alpha \beta} f_{\alpha \beta}$ in the Lagrangian associated with (2.2) (with, in addition, degrees of freedom of the fields) required by dimensionality. The field strengths are

$$
f^{\alpha \beta}=\partial^{\alpha} a^{\beta}-\partial^{\beta} a^{\alpha},
$$

and the current satisfies the conservation law $[10,23,24]$

$$
\partial_{\alpha} j^{\alpha}(x, \tau)=0
$$

integrating over $\tau$ on $(-\infty, \infty)$, and assuming that $j^{5}(x, \tau)$ vanishes at $|\tau| \rightarrow \infty$, one finds that

$$
\partial_{\mu} J^{\mu}(x)=0
$$

where (for some dimensionless $\eta$ ) [17]

$$
J^{\mu}(x)=\eta \int_{-\infty}^{\infty} d \tau j^{\mu}(x, \tau)
$$

We identify this $J^{\mu}(x)$ with the Maxwell conserved current. In [9], for example, this expression occurs with

$$
j^{\mu}(x, \tau)=\dot{x}^{\mu}(\tau) \delta^{4}(x-x(\tau)),
$$

and $\tau$ is identified with the proper time of the particle (an identification which can be made for the motion of a free particle). The conservation of the integrated current then follows from the fact that

$$
\partial_{\mu} j^{\mu}=\dot{x}^{\mu}(\tau) \partial_{\mu} \delta^{4}(x-x(\tau))=-\frac{d}{d \tau} \delta^{4}(x-x(\tau))
$$

a total derivative; we assume that the world line runs to infinity (at least in the time dimension), and therefore, its integral vanishes at the endpoints $[9,27,28]$, in accordance with the discussion above.

As for the Maxwell case, one can write the current formally in five-dimensional form

$$
j^{\alpha}=\dot{x}^{\alpha} \delta^{4}(x(\tau)-x) .
$$

For $\alpha=5$, the factor $\dot{x}^{5}$ is unity, and this component therefore represents the event density in spacetime. 
Integrating the $\mu$-components of (2.4) over $\tau$ (assuming $f^{\mu 5}(x, \tau) \rightarrow 0$ pointwise for $\tau \rightarrow \pm \infty$ ), we obtain the Maxwell equations with the Maxwell charge $e=e_{0} / \eta$ and the Maxwell fields given by

$$
A^{\mu}(x)=\lambda \int_{-\infty}^{\infty} a^{\mu}(x, \tau) d \tau
$$

A Hamiltonian of the form (2.3) without $\tau$ dependence of the fields, and without the $a_{5}$ terms, as written by Stueckelberg $[27,28]$, can be recovered in the limit of the zero mode of the fields (with $a_{5}=0$ ) in a physical state for which this limit is a good approximation, that is, when the Fourier transform of the fields, defined by

$$
a^{\mu}(x, \tau)=\int d s \hat{a}^{\mu}(x, s) e^{-i s \tau}
$$

has support only in a small neighborhood $\Delta s$ of $s=0$. The vector potential then takes on the form $a^{\mu}(x, \tau) \sim \Delta s \hat{a}^{\mu}(x, 0)=(\Delta s / 2 \pi \lambda) A^{\mu}(x)$, and we identify $e=(\Delta s / 2 \pi \lambda) e_{0}$. The zero mode therefore emerges when the inverse correlation length of the field $\Delta s$ is sufficiently small, and then $\eta=2 \pi \lambda / \Delta s$. We remark that in this limit, the fifth equation obtained from (2.4) decouples. The Lorentz force obtained from this Hamiltonian, using the Hamilton equations, coincides with the usual Lorentz force, and, as we have seen, the generalized Maxwell equations reduce to the usual Maxwell equations. The theory therefore contains the usual Maxwell-Lorentz theory in the limit of the zero mode; for this reason, we have called this generalized theory the "pre-Maxwell" theory.

If such a pre-Maxwell theory really underlies the standard Maxwell theory, then there should be some physical mechanism which restricts most observations in the laboratory to be close to the zero mode. For example, in a metal there is a frequency, the plasma frequency, below which there is no transmission of electromagnetic waves. In this case, if the physical universe is imbedded in a medium which does not allow high "frequencies" to pass, the pre-Maxwell theory reduces to the Maxwell theory. Some study has been carried out, for a quite different purpose (of achieving a form of analog gravity), of the properties of the generalized fields in a medium with general dielectric tensor [18]. We will see in the present work that the high level of nonlinearity of this theory in interaction with matter may itself generate an effective reduction to Maxwell-Lorentz theory, with the high-frequency chaotic behavior providing the regularization achieved by models of the type discussed by Rohrlich [20, 21, 22].

We remark that integration over $\tau$ does not bring the generalized Lorentz force into the form of the standard Lorentz force, since it is nonlinear, and a convolution remains. If the resulting convolution is trivial, that is, in the zero mode, the two theories then coincide. Hence, we expect to see dynamical effects in the generalized theory which are not present in the standard Maxwell-Lorentz theory.

Writing the Hamilton equations

$$
\dot{x}^{\mu}=\frac{d x^{\mu}}{d \tau}=\frac{\partial K}{\partial p_{\mu}}, \quad \dot{p}^{\mu}=\frac{d p^{\mu}}{d \tau}=-\frac{\partial K}{d x_{\mu}}
$$


for the Hamiltonian (2.3), we find the generalized Lorentz force

$$
M \ddot{x}^{\mu}=e_{0} f_{\nu}^{\mu} \dot{x}^{\nu}+f_{5}^{\mu}
$$

Multiplying this equation by $\dot{x}_{\mu}$, one obtains

$$
M \dot{x}_{\mu} \ddot{x}^{\mu}=e_{0} \dot{x}_{\mu} f_{5}^{\mu}
$$

this equation therefore does not necessarily lead to the trivial relation between $d s$ and $d \tau$ discussed above in connection with (1.8). The $f_{5}^{\mu}$ term has the effect of moving the particle off-shell (as, in the nonrelativistic case, the energy is altered by the electric field).

We now define

$$
\varepsilon=1+\dot{x}^{\mu} \dot{x}_{\mu}=1-\frac{d s^{2}}{d \tau^{2}}
$$

where $d s^{2}=d t^{2}-d \mathbf{x}^{2}$ is the square of the proper time. Since $\dot{x}^{\mu}=\left(p^{\mu}-e_{0} a^{\mu}\right) / M$, if we interpret $\left(p^{\mu}-e_{0} a^{\mu}\right)\left(p_{\mu}-e_{0} a_{\mu}\right)=-m^{2}$, the gauge invariant particle mass $[10,23,24]$, then

$$
\varepsilon=1-\frac{m^{2}}{M^{2}}
$$

measures the deviation from "mass shell" (on-mass-shell, $d s^{2}=d \tau^{2}$ ).

\section{Derivation of the differential equations for the spacetime orbit with off-shell corrections}

We now review the derivation of the radiation reaction formula in the Stueckelberg formalism (see also [17]). Calculating the self-interaction contribution, one must include the effects of the force acting upon the particle due to its own field $\left(f_{\text {self }}\right)$ in addition to the fields generated by other electromagnetic sources $\left(f_{\text {ext }}\right)$. Therefore, the generalized Lorentz force, using (2.14), takes the form

$$
M \ddot{x}^{\mu}=e_{0} \dot{x}^{\nu} f_{\text {ext }} \stackrel{\mu}{\nu}+e_{0} \dot{x}^{\nu} f_{\text {self }} \stackrel{\mu}{\nu}+e_{0} f_{\text {ext }} \stackrel{\mu}{5}+e_{0} f_{\text {self }} \stackrel{\mu}{5},
$$

where the dynamical derivatives (dot) are with respect to the universal time $\tau$, and the fields are evaluated on the event's trajectory. Multiplying (3.1) by $\dot{x}_{\mu}$, we get the projected equation (2.16) in the form

$$
\frac{M}{2} \dot{\varepsilon}=e_{0} \dot{x}_{\mu} f_{\text {ext }}^{\mu}+e_{0} \dot{x}_{\mu} f_{\text {self }} \stackrel{\mu}{5}
$$


The field generated by the current is given by the pre-Maxwell equations (2.4), and choosing for it the generalized Lorentz gauge $\partial_{\alpha} a^{\alpha}=0$, we get

$$
\lambda \partial_{\alpha} \partial^{\alpha} a^{\beta}(x, \tau)=\lambda\left(\sigma \partial_{\tau}^{2}-\partial_{t}^{2}+\nabla^{2}\right) a^{\beta}=-e_{0} j^{\beta}(x, \tau),
$$

where $\sigma= \pm 1$ corresponds to the possible choices of metric for the symmetry $\mathrm{O}(4,1)$ or $\mathrm{O}(3,2)$ of the homogeneous field equations.

Green's functions for (3.3) can be constructed from the inverse Fourier transform

$$
G(x, \tau)=\frac{1}{(2 \pi)^{5}} \int d^{4} k d \kappa \frac{e^{i\left(k^{\mu} x_{\mu}+\sigma \kappa \tau\right)}}{k_{\mu} k^{\mu}+\sigma \kappa^{2}} .
$$

Integrating this expression over all $\tau$ gives Green's function for the standard Maxwell field. Assuming that the radiation reaction acts causally in $\tau$, we will use here the $\tau$-retarded Green's function. In his calculation of the radiation corrections to the Lorentz force, Dirac used the difference between advanced and retarded Green's functions in order to cancel the singularities that they contain. One can, alternatively, use the retarded Green's function and "renormalize" the mass in order to eliminate the singularity [25]. In this analysis, we follow the latter procedure.

The $\tau$-retarded Green's function [17] is given by multiplying the principal part of the integral equation (3.4) by $\theta(\tau)$. Carrying out the integrations (on a complex contour in $\kappa$; we consider the case $\sigma=+1$ in the following), one finds (this Green's function differs from the $t$-retarded Green's function, constructed on a complex contour in $k^{0}$ )

$$
G(x, \tau)=\frac{2 \theta(\tau)}{(2 \pi)^{3}} \begin{cases}\frac{\tan ^{-1}\left(\sqrt{-x^{2}-\tau^{2}} / \tau\right)}{\left(-x^{2}-\tau^{2}\right)^{3 / 2}}-\frac{\tau}{x^{2}\left(x^{2}+\tau^{2}\right)}, & x^{2}+\tau^{2}<0, \\ \frac{1}{2} \frac{1}{\left(\tau^{2}+x^{2}\right)^{3 / 2}} \ln \left|\frac{\tau-\sqrt{\tau^{2}+x^{2}}}{\tau+\sqrt{\tau^{2}+x^{2}}}\right|-\frac{\tau}{x^{2}\left(\tau^{2}+x^{2}\right)}, & x^{2}+\tau^{2}>0,\end{cases}
$$

where we have written $x^{2} \equiv x^{\mu} x_{\mu}$.

With the help of this Green's function, the solutions of (3.3) for the self-fields (substituting the current from (2.11)) are

$$
\begin{aligned}
a_{\text {self }}^{\mu}(x, \tau) & =\frac{e_{0}}{\lambda} \int d^{4} x^{\prime} d \tau^{\prime} G\left(x-x^{\prime}, \tau-\tau^{\prime}\right) \dot{x}^{\mu}\left(\tau^{\prime}\right) \delta^{4}\left(x^{\prime}-x\left(\tau^{\prime}\right)\right) \\
& =\frac{e_{0}}{\lambda} \int d \tau^{\prime} \dot{x}^{\mu}\left(\tau^{\prime}\right) G\left(x-x\left(\tau^{\prime}\right), \tau-\tau^{\prime}\right), \\
a_{\text {self }}^{5}(x, \tau) & =\frac{e_{0}}{\lambda} \int d \tau^{\prime} G\left(x-x\left(\tau^{\prime}\right), \tau-\tau^{\prime}\right) .
\end{aligned}
$$

Green's function is written as a scalar, acting in the same way on all five components of the source $j^{\alpha}$; to assure that the resulting field is in Lorentz gauge, however, it should be written as a five-by-five matrix, with the factor $\delta_{\beta}^{\alpha}-k^{\alpha} k_{\beta} / k^{2}\left(k_{5}=\kappa\right)$ included in the 
integrand. Since we compute only the gauge invariant field strengths here, this extra term will not influence any of the results. It then follows that the generalized Lorentz force for the self-action (the force of the fields generated by the world line on a point $x^{\mu}(\tau)$ of the trajectory), along with the effect of external fields, is

$$
\begin{aligned}
M \ddot{x}^{\mu}= & \left.\frac{e_{0}^{2}}{\lambda} \int d \tau^{\prime}\left(\dot{x}^{\nu}(\tau) \dot{x}_{\nu}\left(\tau^{\prime}\right) \partial^{\mu}-\dot{x}^{\nu}(\tau) \dot{x}^{\mu}\left(\tau^{\prime}\right) \partial_{\nu}\right) G\left(x-x\left(\tau^{\prime}\right)\right)\right|_{x=x(\tau)} \\
& +\left.\frac{e_{0}^{2}}{\lambda} \int d \tau^{\prime}\left(\partial^{\mu}-\dot{x}^{\mu}\left(\tau^{\prime}\right) \partial_{\tau}\right) G\left(x-x\left(\tau^{\prime}\right)\right)\right|_{x=x(\tau)} \\
& +e_{0}\left(f_{\text {ext }}{ }_{\nu}^{\mu} \dot{x}^{\nu}+f_{\text {ext }} \stackrel{\mu}{5}\right) .
\end{aligned}
$$

We define $u \equiv\left(x_{\mu}(\tau)-x_{\mu}\left(\tau^{\prime}\right)\right)\left(x^{\mu}(\tau)-x^{\mu}\left(\tau^{\prime}\right)\right)$ so that

$$
\partial_{\mu}=2\left(x_{\mu}(\tau)-x_{\mu}\left(\tau^{\prime}\right)\right) \frac{\partial}{\partial u}
$$

Equation (3.7) then becomes

$$
\begin{aligned}
M \ddot{x}^{\mu}= & 2 \frac{e_{0}^{2}}{\lambda} \int d \tau^{\prime}\left\{\dot{x}^{\nu}(\tau) \dot{x}_{\nu}\left(\tau^{\prime}\right)\left(x^{\mu}(\tau)-x^{\mu}\left(\tau^{\prime}\right)\right)\right. \\
& \left.\quad-\dot{x}^{\nu}(\tau) \dot{x}^{\mu}\left(\tau^{\prime}\right)\left(x_{\nu}(\tau)-x_{\nu}\left(\tau^{\prime}\right)\right)\right\}\left.\frac{\partial}{\partial u} G\left(x-x\left(\tau^{\prime}\right), \tau-\tau^{\prime}\right)\right|_{x=x(\tau)} \\
& +\left.\frac{e_{0}^{2}}{\lambda} \int d \tau^{\prime}\left(2\left(x^{\mu}(\tau)-x^{\mu}\left(\tau^{\prime}\right)\right) \frac{\partial}{\partial u}-\dot{x}^{\mu}\left(\tau^{\prime}\right) \partial_{\tau}\right) G\left(x-x\left(\tau^{\prime}\right), \tau-\tau^{\prime}\right)\right|_{x=x(\tau)} \\
& +e_{0}\left(f_{\text {ext }}^{\mu} \dot{x}^{\nu}+f_{\text {ext }}{ }_{5}^{\mu}\right) .
\end{aligned}
$$

In the self-interaction problem where $\tau \rightarrow \tau^{\prime}, x^{\mu}\left(\tau^{\prime}\right)-x^{\mu}(\tau) \rightarrow 0$, Green's function is very divergent. Therefore, one can expand all expressions in $\tau^{\prime \prime}=\tau-\tau^{\prime}$ assuming that the dominant contribution is from the neighborhood of small $\tau^{\prime \prime}$. The divergent terms are later absorbed into the mass and charge definitions leading to renormalization (effective mass and charge). Expanding the integrands in Taylor series around the most singular point $\tau^{\prime}=\tau$ and keeping the lowest-order terms, the variable $u$ reduces to

$$
u \cong \dot{x}^{\mu} \dot{x}_{\mu} \tau^{\prime \prime 2}-\dot{x}_{\mu} \ddot{x}^{\mu} \tau^{\prime \prime 3}+\frac{1}{3} \dot{x}_{\mu} \dddot{x}^{\mu} \tau^{\prime \prime 4}+\frac{1}{4} \ddot{x}_{\mu} \ddot{x}^{\mu} \tau^{\prime \prime 4}
$$

(In [17], we considered $u \cong \dot{x}^{\mu} \dot{x}_{\mu} \tau^{\prime \prime 2}$ omitting several possibly significant terms. The corrected equations here contain these additional terms.)

We now recall the definition of the off-shell deviation $\varepsilon$ given in (2.17), along with its derivatives:

$$
\dot{x}_{\mu} \dot{x}^{\mu}=-1+\varepsilon, \quad \dot{x}_{\mu} \ddot{x}^{\mu}=\frac{1}{2} \dot{\varepsilon}, \quad \dot{x}_{\mu} \ddot{x}^{\mu}+\ddot{x}_{\mu} \ddot{x}^{\mu}=\frac{1}{2} \ddot{\varepsilon} .
$$


Next, we define

$$
w \equiv \frac{1}{12} \dot{x}_{\mu} \dddot{x}^{\mu}+\frac{1}{8} \ddot{\varepsilon}, \quad \Delta \equiv-\frac{1}{2} \dot{\varepsilon} \tau^{\prime \prime}+w \tau^{\prime \prime 2} .
$$

Using these definitions along with those of (3.10) and (3.11), we find

$$
\frac{u+\tau^{\prime \prime 2}}{\tau^{\prime \prime 2}} \cong \varepsilon+\Delta
$$

We then expand Green's function to leading orders:

$$
\begin{aligned}
\frac{\partial G}{\partial u} \cong & \frac{\theta\left(\tau^{\prime \prime}\right) f_{1}(\epsilon+\Delta)}{(2 \pi)^{3} \tau^{\prime \prime}}=\frac{\theta\left(\tau^{\prime \prime}\right)}{(2 \pi)^{3}}\left[\frac{f_{1}(\varepsilon)}{\tau^{\prime \prime}}-\frac{\dot{\varepsilon} f_{1}^{\prime}(\varepsilon)}{2 \tau^{\prime \prime}}+\left(w f_{1}^{\prime}(\varepsilon)+\frac{1}{8} \dot{\varepsilon}^{2} f_{1}^{\prime \prime}(\varepsilon)\right) \frac{1}{\tau^{\prime \prime 3}}\right], \\
\frac{\partial G}{\partial \tau^{\prime \prime}} \cong & \frac{2 \theta\left(\tau^{\prime \prime}\right) f_{2}(\epsilon+\Delta)}{(2 \pi)^{3} \tau^{\prime \prime}}+\frac{2 \delta\left(\tau^{\prime \prime}\right) f_{3}(\epsilon+\Delta)}{(2 \pi)^{3} \tau^{\prime \prime}} \\
= & \frac{2 \theta\left(\tau^{\prime \prime}\right)}{(2 \pi)^{3}}\left[\frac{f_{2}(\varepsilon)}{\tau^{\prime \prime}}-\frac{\dot{\varepsilon} f_{2}^{\prime}(\varepsilon)}{2 \tau^{\prime \prime 3}}+\left(w f_{2}^{\prime}(\varepsilon)+\frac{1}{8} \dot{\varepsilon}^{2} f_{2}^{\prime \prime}(\varepsilon)\right) \frac{1}{\tau^{\prime \prime 2}}\right] \\
& +\frac{2 \delta\left(\tau^{\prime \prime}\right)}{(2 \pi)^{3}}\left[\frac{f_{3}(\varepsilon)}{\tau^{\prime \prime 3}}-\frac{\dot{\varepsilon} f_{3}^{\prime}(\varepsilon)}{2 \tau^{\prime \prime 2}}+\left(w f_{3}^{\prime}(\varepsilon)+\frac{1}{8} \dot{\varepsilon}^{2} f_{3}^{\prime \prime}(\varepsilon)\right) \frac{1}{\tau^{\prime \prime}}\right],
\end{aligned}
$$

where $f^{\prime} \equiv d f / d \varepsilon$, and for $\varepsilon<0$,

$$
\begin{aligned}
& f_{1}(\varepsilon)=\frac{3 \tan ^{-1}(\sqrt{-\varepsilon})}{(-\varepsilon)^{5 / 2}}-\frac{3}{\varepsilon^{2}(1-\varepsilon)}+\frac{2}{\varepsilon(1-\varepsilon)^{2}}, \\
& f_{2}(\varepsilon)=\frac{3 \tan ^{-1}(\sqrt{-\varepsilon})}{(-\varepsilon)^{5 / 2}}-\frac{1}{\varepsilon^{2}}-\frac{2-\varepsilon}{\varepsilon^{2}(1-\varepsilon)}, \\
& f_{3}(\varepsilon)=\frac{\tan ^{-1}(\sqrt{-\varepsilon})}{(-\varepsilon)^{3 / 2}}+\frac{1}{\varepsilon(1-\varepsilon)} .
\end{aligned}
$$

For $\varepsilon>0$,

$$
\begin{aligned}
& f_{1}(\varepsilon)=\frac{(3 / 2) \ln |(1+\sqrt{\varepsilon}) /(1-\sqrt{\varepsilon})|}{(\varepsilon)^{5 / 2}}-\frac{3}{\varepsilon^{2}(1-\varepsilon)}+\frac{2}{\varepsilon(1-\varepsilon)^{2}}, \\
& f_{2}(\varepsilon)=\frac{(3 / 2) \ln |(1+\sqrt{\varepsilon}) /(1-\sqrt{\varepsilon})|}{(\varepsilon)^{5 / 2}}-\frac{1}{\varepsilon^{2}}-\frac{2-\varepsilon}{\varepsilon^{2}(1-\varepsilon)}, \\
& f_{3}(\varepsilon)=-\frac{(1 / 2) \ln |(1+\sqrt{\varepsilon}) /(1-\sqrt{\varepsilon})|}{\varepsilon^{3 / 2}}+\frac{1}{\varepsilon(1-\varepsilon)} .
\end{aligned}
$$


For either sign of $\varepsilon$, when $\varepsilon \sim 0$,

$$
\begin{aligned}
& f_{1}(\varepsilon) \sim \frac{8}{5}+\frac{24}{7} \varepsilon+\frac{16}{3} \varepsilon^{2}+O\left(\varepsilon^{3}\right), \\
& f_{2}(\varepsilon) \sim-\frac{2}{5}-\frac{4}{7} \varepsilon-\frac{2}{3} \varepsilon^{2}+O\left(\varepsilon^{3}\right), \\
& f_{3}(\varepsilon) \sim \frac{2}{3}+\frac{4}{5} \varepsilon+\frac{6}{7} \varepsilon^{2}+O\left(\varepsilon^{3}\right) .
\end{aligned}
$$

One sees that the derivatives in (3.14) have no singularity in $\varepsilon$ at $\varepsilon=0$.

From (3.6), we have

$$
\begin{aligned}
f_{\text {self }}^{\mu}{ }_{5}(x(\tau), \tau)=e \int & \left(2\left(x^{\mu}(\tau)-x^{\mu}\left(\tau^{\prime}\right)\right) \frac{\partial}{\partial u}-\dot{x}^{\mu}\left(\tau^{\prime}\right) \partial_{\tau}\right) \\
& \times\left. G\left(x-x\left(\tau^{\prime}\right), \tau-\tau^{\prime}\right)\right|_{x=x(\tau)} d \tau^{\prime} .
\end{aligned}
$$

We then expand $x^{\mu}(\tau)-x^{\mu}\left(\tau^{\prime}\right)$ and $\dot{x}^{\mu}(\tau)-\dot{x}^{\mu}\left(\tau^{\prime}\right)$ in power series in $\tau^{\prime \prime}$, and write the integrals formally with infinite limits.

Substituting (3.18) into (3.2), we obtain (note that $x^{\mu}$ and its derivatives are evaluated at the point $\tau$, and are not subject to the $\tau^{\prime \prime}$ integration), after integrating by parts using $\delta\left(\tau^{\prime \prime}\right)=\left(\partial / \partial \tau^{\prime \prime}\right) \theta\left(\tau^{\prime \prime}\right)$,

$$
\begin{aligned}
\frac{M}{2} \dot{\varepsilon}=\frac{2 e_{0}^{2}}{\lambda(2 \pi)^{3}} \int_{-\infty}^{\infty} d \tau^{\prime \prime}\{ & \frac{g_{1}}{\tau^{\prime \prime 4}}(\varepsilon-1)-\frac{g_{2}}{\tau^{\prime \prime 3}} \frac{\dot{\varepsilon}}{2}+\frac{g_{3}}{\tau^{\prime \prime 2}} \dot{x}_{\nu} \dddot{x}^{\nu}-\frac{h_{1}}{\tau^{\prime \prime 3}}(\varepsilon-1) \dot{\varepsilon}+\frac{h_{2}}{2 \tau^{\prime \prime 2}} \dot{\varepsilon}^{2} \\
& \left.+\frac{h_{3}}{\tau^{\prime \prime 2}}(\varepsilon-1) w+\frac{h_{4}}{8 \tau^{\prime \prime 2}} \dot{\varepsilon}^{2}(\varepsilon-1)\right\} \theta\left(\tau^{\prime \prime}\right)+e_{0} \dot{x}_{\mu} f_{\text {ext }}^{\mu},
\end{aligned}
$$

where we have defined

$$
\begin{gathered}
g_{1}=f_{1}-f_{2}-3 f_{3}, \quad g_{2}=\frac{1}{2} f_{1}-f_{2}-2 f_{3}, \quad g_{3}=\frac{1}{6} f_{1}-\frac{1}{2} f_{2}-\frac{1}{2} f_{3}, \\
h_{1}=\frac{1}{2} f_{1}^{\prime}-\frac{1}{2} f_{2}^{\prime}-f_{3}^{\prime}, \quad h_{2}=\frac{1}{4} f_{1}^{\prime}-\frac{1}{2} f_{2}^{\prime}-\frac{1}{2} f_{3}^{\prime}, \\
h_{3}=\left(f_{1}^{\prime}-f_{2}^{\prime}-f_{3}^{\prime}\right), \quad h_{4}=f_{1}^{\prime \prime}-f_{2}^{\prime \prime}-f_{3}^{\prime \prime} .
\end{gathered}
$$

The integrals are divergent at the lower bound $\tau^{\prime \prime}=0$ imposed by the $\theta$-function; we therefore take these integrals to a cutoff $\mu>0$. Equation (3.19) then becomes

$$
\begin{gathered}
\frac{M}{2} \dot{\varepsilon}=\frac{2 e_{0}^{2}}{\lambda(2 \pi)^{3}}\left\{\frac{g_{1}}{3 \mu^{3}}(\varepsilon-1)-\frac{g_{2}}{4 \mu^{2}} \dot{\varepsilon}+\frac{g_{3}}{\mu} \dot{x}_{\nu} \ddot{x}^{\nu}-\frac{h_{1}}{2 \mu^{2}}(\varepsilon-1) \dot{\varepsilon}+\frac{h_{2}}{2 \mu} \dot{\varepsilon}^{2}+\frac{h_{3}}{\mu}(\varepsilon-1) w\right. \\
\left.+\frac{h_{4}}{8 \mu} \dot{\varepsilon}^{2}(\varepsilon-1)\right\} \theta\left(\tau^{\prime \prime}\right)+e_{0} \dot{x}_{\mu} f_{\text {ext }} \frac{\mu}{5} .
\end{gathered}
$$


Following a similar procedure, we obtain from (3.7)

$$
\begin{aligned}
M \ddot{x}^{\mu}= & \frac{2 e_{0}^{2}}{\lambda(2 \pi)^{3}}\left\{-\frac{1}{2}\left((1-\varepsilon) \ddot{x}^{\mu}+\frac{\dot{\varepsilon}}{2} \dot{x}^{\mu}\right)\left(\frac{f_{1}}{2 \mu^{2}}-\frac{\dot{\varepsilon} f_{1}^{\prime}}{2 \mu}\right)+\frac{f_{1}}{3 \mu}\left(\dot{x}_{\nu} \dddot{x}^{\nu} \dot{x}^{\mu}+(1-\varepsilon) \dddot{x}^{\mu}\right)\right. \\
& \left.+\frac{g_{1}}{3 \mu^{3}} \dot{x}^{\mu}-\frac{g_{2}}{2 \mu^{2}} \ddot{x}^{\mu}+\frac{g_{3}}{\mu} \dddot{x}^{\mu}-\frac{h_{1} \dot{\varepsilon}}{2 \mu^{2}} \dot{x}^{\mu}+\frac{h_{2} \dot{\varepsilon}}{\mu} \ddot{x}^{\mu}+\frac{h_{3} w}{\mu} \dot{x}^{\mu}+\frac{h_{4} \dot{\varepsilon}^{2}}{8 \mu} \dot{x}^{\mu}\right\} \\
& +e_{0}\left(f_{\text {ext }}^{\mu} \dot{x}^{\nu}+f_{\text {ext }}^{\mu}\right) .
\end{aligned}
$$

Substituting (3.21) for the coefficients of the $\dot{x}^{\mu}$ terms in the second line of (3.22), we find

$$
\begin{aligned}
M(\varepsilon, \dot{\varepsilon}) \ddot{x}^{\mu}= & -\frac{1}{2} \frac{M(\varepsilon, \dot{\varepsilon})}{(1-\varepsilon)} \dot{\varepsilon} \dot{x}^{\mu}+\frac{2 e_{0}^{2}}{\lambda(2 \pi)^{3} \mu} F(\varepsilon)\left\{\dddot{x}^{\mu}+\frac{1}{1-\varepsilon} \dot{x}_{\nu} \dddot{x}^{\nu} \dot{x}^{\mu}\right\} \\
& +\frac{e_{0} \dot{x}^{\mu} \dot{x}_{\nu} f_{\text {ext }}{ }^{\nu}}{1-\varepsilon}+e_{0} f_{\text {ext }}{ }_{\nu}^{\mu} \dot{x}^{\nu}+e_{0} f_{\text {ext }} \stackrel{\mu}{5},
\end{aligned}
$$

where

$$
F(\varepsilon)=\frac{f_{1}}{3}(1-\varepsilon)+g_{3}
$$

Here, the coefficients of $\ddot{x}^{\mu}$ have been grouped formally into a renormalized (off-shell) mass term, defined (as done in the standard radiation reaction problem) as

$$
M(\varepsilon, \dot{\varepsilon})=M+\frac{e^{2}}{2 \mu}\left[\frac{f_{1}(1-\varepsilon)}{2}+g_{2}\right]-e^{2}\left[\frac{1}{4} f_{1}^{\prime}(1-\varepsilon)+h_{2}\right] \dot{\varepsilon},
$$

where, as we will see below,

$$
e^{2}=\frac{2 e_{0}^{2}}{\lambda(2 \pi)^{3} \mu}
$$

can be identified with the Maxwell charge by studying the on-shell limit.

We remark that one can change variables, with the help of (2.17) (here, for simplicity, assuming $\varepsilon<1$ ), to obtain a differential equation in which all derivatives with respect to $\tau$ are replaced by derivatives with respect to the proper time $s$. The coefficient of the second derivative of $x^{\mu}$ with respect to $s$, and "effective mass" for the proper-time equation, is then given by

$$
\begin{aligned}
M_{S}(\varepsilon, \dot{\varepsilon})=\frac{2}{3 F(\varepsilon)(1-\varepsilon)}\{ & M+\frac{e^{2}}{2 c^{3} \mu}\left[f_{1}(1-\varepsilon) 2+g_{2}\right] \\
& \left.-\frac{e^{2}}{c^{3}}\left[\frac{1}{4} f_{1}(1-\varepsilon)+h_{2}+\frac{3}{2} F(\varepsilon) \sqrt{1-\varepsilon}\right] \dot{\varepsilon}\right\} .
\end{aligned}
$$


Note that the renormalized mass depends on $\varepsilon(\tau)$; for this quantity to act as a mass, $\varepsilon$ must be slowly varying on some interval on the orbit of the evolution compared to all other motions. The computer analysis we give below indeed shows that there are large intervals of almost constant $\varepsilon$. In case, as at some points, $\varepsilon$ may be rapidly varying, one may consider the definition (3.25) as formal; clearly, however, if $M(\varepsilon, \dot{\varepsilon})$ is large, $\ddot{x}^{\mu}$ will be suppressed (e.g., for $\varepsilon$ close to unity, where $M_{S}(\varepsilon, \dot{\varepsilon})$ goes as $\dot{\varepsilon} /(1-\varepsilon)^{2}$ and $M(\varepsilon, \dot{\varepsilon})$ as $\dot{\varepsilon} /(1-\varepsilon)^{3}$; note that $F(\varepsilon)$ goes as $\left.(1-\varepsilon)^{-3}\right)$.

We now obtain, from (3.23),

$$
\begin{aligned}
M(\varepsilon, \dot{\varepsilon}) \ddot{x}^{\mu}= & -\frac{1}{2} \frac{M(\varepsilon, \dot{\varepsilon})}{(1-\varepsilon)} \dot{\varepsilon} \dot{x}^{\mu}+F(\varepsilon) e^{2}\left\{\dddot{x}^{\mu}+\frac{1}{1-\varepsilon} \dot{x}_{\nu} \dddot{x}^{\nu} \dot{x}^{\mu}\right\} \\
& +e_{0} f_{\operatorname{ext}_{v}}^{\mu} \dot{x}^{\nu}+e_{0}\left(\frac{\dot{x}^{\mu} \dot{x}_{v}}{1-\varepsilon}+\delta_{v}^{\mu}\right) f_{\operatorname{ext}_{5} .}^{\nu}
\end{aligned}
$$

We remark that when one multiplies this equation by $\dot{x}_{\mu}$, it becomes an identity (all of

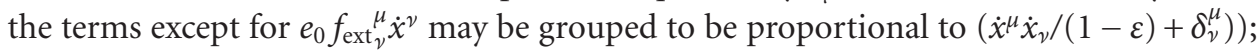
one must use (3.21) to compute the off-shell mass shift $\varepsilon$ corresponding to the longitudinal degree of freedom in the direction of the four velocity of the particle. Equation (3.28) determines the motion orthogonal to the four velocity. Equations (3.21) and (3.28) are the fundamental dynamical equations governing the off-shell orbit.

We remark that as in [17], it can be shown that (3.28) reduces to the ordinary (Abraham-Lorentz-Dirac) radiation reaction formula for small, slowly changing $\varepsilon$ and that no instability, no radiation, and no acceleration of the electron occurs when it is on-shell. There is therefore no "runaway solution" for the exact mass-shell limit of this theory; the unstable Dirac result is approximate for $\varepsilon$ close to, but not precisely, zero.

\section{The $\varepsilon$ evolution}

We now derive an equation for the evolution of the off-shell deviation $\varepsilon$ when the external field is removed. We then use this equation to prove that a fixed mass shell is consistent only if the particle is not accelerating, and therefore no runaway solution occurs. Using the definitions

$$
\begin{gathered}
F_{1}(\varepsilon)=\frac{g_{1}(\varepsilon-1)}{3 \mu^{2}}, \quad F_{2}(\varepsilon)=\frac{g_{2}-2(\varepsilon-1) h_{1}}{4 \mu} \\
F_{3}(\varepsilon)=g_{3}+\frac{1}{12}(\varepsilon-1) h_{3}, \quad F_{4}(\varepsilon)=\frac{1}{2} h_{2}+\frac{1}{8}(\varepsilon-1) h_{4} \\
F_{5}(\varepsilon)=\frac{1}{8}(1-\varepsilon) h_{3}
\end{gathered}
$$

in (3.21), in the absence of external fields, we write

$$
\dot{x}_{\mu} \dddot{x}^{\mu}=\frac{1}{F_{3}(\varepsilon)}\left\{\frac{M}{2 e^{2}} \dot{\varepsilon}-F_{1}(\varepsilon)+F_{2}(\varepsilon) \dot{\varepsilon}-F_{4}(\varepsilon) \dot{\varepsilon}^{2}-F_{5}(\varepsilon) \ddot{\varepsilon}\right\} .
$$


Differentiating with respect to $\tau$, we find

$$
\begin{aligned}
\dot{x}_{\mu} \ddot{x}^{\mu}+\ddot{x}_{\mu} \dddot{x}^{\mu}= & \frac{1}{F_{3}}\left\{F_{2}^{\prime} \dot{\varepsilon}^{2}+\ddot{\varepsilon}\left(\frac{M}{2 e^{2}}+F_{2}\right)-F_{1}^{\prime} \dot{\varepsilon}-F_{4}^{\prime}(\varepsilon) \dot{\varepsilon}^{3}-\left(2 F_{4}(\varepsilon)+-F_{5}^{\prime}(\varepsilon)\right) \dot{\varepsilon} \ddot{\varepsilon}-F_{5} \dddot{\varepsilon}\right\} \\
& -\frac{F_{3}^{\prime}}{F_{3}{ }^{2}}\left\{F_{2}+\frac{M}{2 e^{2}} \dot{\varepsilon}-F_{1}-F_{4}(\varepsilon) \dot{\varepsilon}^{2}-F_{5}(\varepsilon) \ddot{\varepsilon}\right\} \dot{\varepsilon} \\
\equiv & H-\frac{F_{5}}{F_{3}} \dddot{\varepsilon} .
\end{aligned}
$$

Together with

$$
\dot{x}_{\mu} \dddot{x}^{\mu}+3 \ddot{x}_{\mu} \dddot{x}^{\mu}=\frac{1}{2} \dddot{\varepsilon},
$$

which is the $\tau$ derivative of the last equation in (3.10), one finds from (4.3) that

$$
\ddot{x}_{\mu} \dddot{x}^{\mu}=\left(\frac{1}{4}+\frac{F_{5}}{2 F_{3}}(\varepsilon)\right) \dddot{\varepsilon}-\frac{1}{2} H(\varepsilon, \dot{\varepsilon}, \ddot{\varepsilon}) .
$$

Multiplying (3.28) by $\ddot{x}_{\mu}$ (with no external fields) and using (4.2) and (4.5), we obtain

$$
\left(1+2 \frac{F_{5}}{F_{3}}\right) \dddot{\varepsilon}-A(\varepsilon) \ddot{\varepsilon}+B(\varepsilon) \dot{\varepsilon}^{2}+C(\varepsilon) \dot{\varepsilon}-D(\varepsilon)+E(\varepsilon) \dot{\varepsilon}^{3}+I(\varepsilon) \ddot{\varepsilon} \ddot{\varepsilon}=0,
$$

where

$$
\begin{gathered}
A=\frac{2}{F_{3}}\left(\frac{M}{2 e^{2}}+F_{2}\right)+\frac{2 M(\varepsilon)}{e^{2} F(\varepsilon)}-\frac{4 M(\varepsilon) F_{5}}{2 e^{2} F(\varepsilon)}, \\
B=\frac{2 F_{3}^{\prime}}{F_{3}^{2}}\left(F_{2}-\frac{M}{2 e^{2}}\right)-\frac{2 F_{2}^{\prime}}{F_{3}}+\frac{2}{1-\varepsilon} \frac{1}{F_{3}}\left(\frac{M}{2 e^{2}}+F_{2}\right)-\frac{M(\varepsilon)}{e^{2} F(\varepsilon)} \frac{1}{1-\varepsilon}, \\
C=\frac{4 M(\varepsilon)}{e^{2} F(\varepsilon)} \frac{1}{F_{3}}\left(\frac{M}{2 e^{2}}+F_{2}\right)-\frac{2}{F_{3}^{2}} F_{1} F_{3}^{\prime}-\frac{2 F_{1}}{(1-\varepsilon) F_{3}}+\frac{2}{F_{3}} F_{1}^{\prime}, \\
D=\frac{4 M(\varepsilon)}{e^{2} F(\varepsilon)} \frac{F_{1}}{F_{3}}, \\
I=2\left(\frac{F_{5}^{\prime}}{F_{3}}+2 \frac{F_{4}}{F_{3}}-\frac{F_{5} F_{3}^{\prime}}{F_{3}^{2}}\right)-\frac{2 F_{5}}{(1-\varepsilon) F_{3}} .
\end{gathered}
$$




\section{Dynamical behavior of system}

In this section, we present results of a preliminary study of the dynamical behavior of the system. The four orbit equations pose a very heavy computational problem, which we will treat in a later publication. We remark here, however, that the quantities $d \mathbf{x} / d \tau$ and $d t / d \tau$ appear to rise rapidly, but the ratio corresponding to the observed velocity $d \mathbf{x} / d t$ actually falls, indicating that what would be observed is a dissipative effect.

We will concentrate here on the evolution of the off-shell mass correction $\varepsilon$, and show that there is a highly complex dynamical behavior showing strong evidence of chaos.

It appears that $\varepsilon$ reaches values close to (but less than) unity, and there exhibits very complex behavior with large fluctuations. The effective mass $M(\varepsilon, \dot{\varepsilon})$ therefore appears to reach a macroscopically steady value, for $\varepsilon$ apparently a little less than unity. In this neighborhood, it is easy to see from expression (3.25) that the effective mass itself becomes very large, as $\dot{\varepsilon} /(1-\varepsilon)^{3}$, and therefore the effect of the Lorentz forces in producing acceleration becomes small.

The third-order equation of $\varepsilon,(4.6)$, can be written as a set of three first-order equations of the form

$$
\frac{d \gamma}{d t}=H(\gamma)
$$

where $\gamma=\gamma 1, \gamma 2, \gamma 3=\varepsilon, \dot{\varepsilon}, \ddot{\varepsilon}$. We have obtained numerical solutions over a range of variations of $\tau$. In Figure 5.1, we show, on a logarithmic scale, these three functions; one sees rapid fluctuations in $\gamma 2$ and $\gamma 3$ and large variations in $\gamma 1$. The action of what appears to be an attractor occurs on these graphs in the neighborhood of $\tau=0.002$ (time steps were taken to be of the order of $10^{-6}$ ). The time that the system remains on this attractor, in our calculation about four times this interval, covers about three cycles. The characteristics of the orbits in phase space are very complex, reflecting an evolution of the nature of the attractor.

The dynamical significance of these functions is most clearly seen in the phase space plots. In Figure 5.2, we show $\ddot{\varepsilon}=\gamma 3$ versus $\dot{\varepsilon}=\gamma 2$. This figure shows the approach to the apparent attractor inducing motion beginning at the lower convex portion of the unsymmetrical orbit which then turns back in a characteristic way to reach the last point visible on this graph. We then continue to examine the motion on a larger scale, where we show, in Figure 5.3, that this endpoint opens to a larger and more symmetric pattern. The orbit then continues, as shown in Figure 5.4, to a third loop, much smaller, which then reaches a structure that is significantly different. The end region of this cycle is shown on a larger scale in Figure 5.5, where a small cycle terminates in oscillations that appear to become very rapid as $\varepsilon$ approaches unity. The coefficients in the differential equation become very large in this neighborhood.

In Figure 5.6, we see the approach to the attractor in the graph of $\dot{\varepsilon}=\gamma 2$ versus $\varepsilon=\gamma 1$; this curve enters a region of folding, which develops to a loop shown in Figure 5.7(a), and in Figure 5.7(b), on a larger scale, where we had to terminate the calculation due to limitations of our computer. 


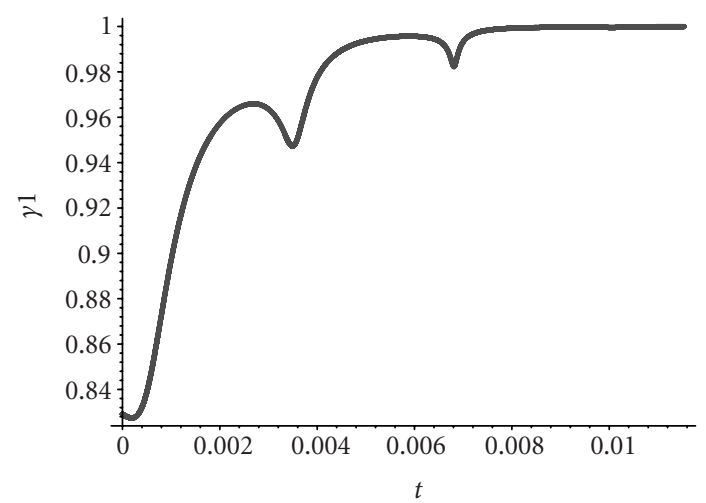

(a)

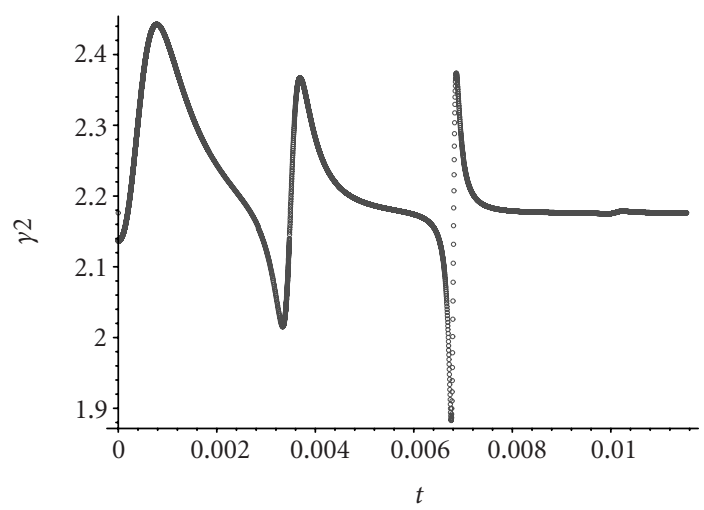

(b)

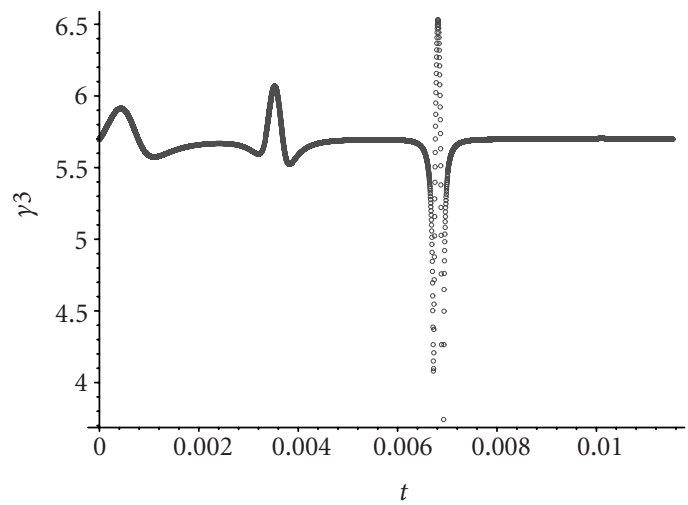

(c)

Figure 5.1. $\varepsilon, \dot{\varepsilon}$, and $\ddot{\varepsilon}$ as functions of $\tau$. These are called $\gamma 1, \gamma 2$, and $\gamma 3$, respectively, on the figures. To make (b) and (c) convenient to represent, we have added 150 to the values of (b) and $5 \times 10^{3}$ to (c), assuring positivity (and value greater than unity) in our data range, and taken the logarithm base 10 . Recursive, approximate quasiperiodic behavior is clearly visible in the variables $\dot{\varepsilon}, \ddot{\varepsilon}$. 


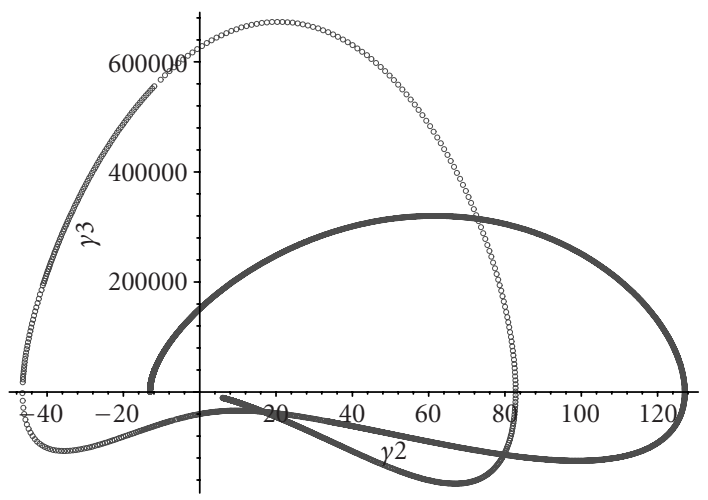

Figure 5.2. The orbit as it enters the region of the attractor, shown in the space of $\dot{\varepsilon}, \ddot{\varepsilon}$, showing the transition from the initial conditions to the strange attractor. In $\tau$, the curve starts on the $\dot{\varepsilon}$ axis to the right of the origin, and ends at the lower left of the origin.

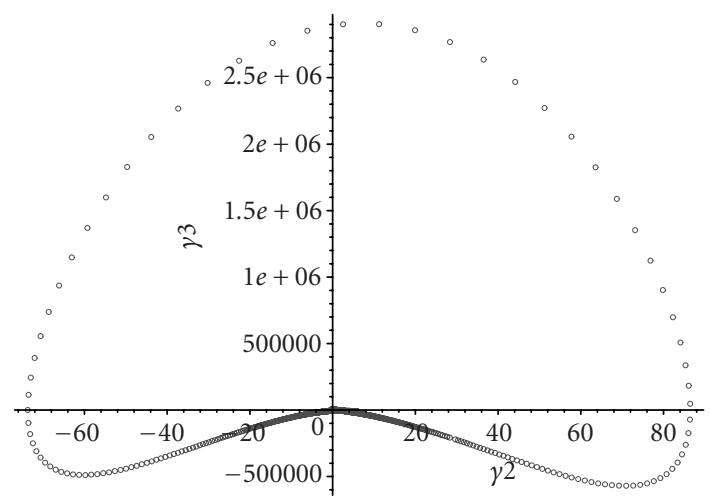

Figure 5.3. This curve continues the evolution within the attractor, starting in the neighborhood of the origin, winding up and around to the right, and terminating almost at the same point (a little above), coming in from the right.

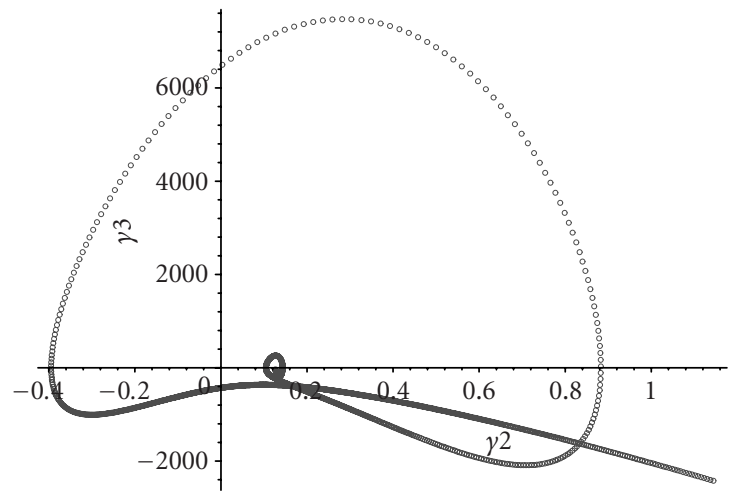

Figure 5.4. This curve is a magnification of the motion in the neighborhood of the endpoint of Figure 5.3, starting below the axis and moving to the left. It makes another loop and ends in a smaller pattern. 


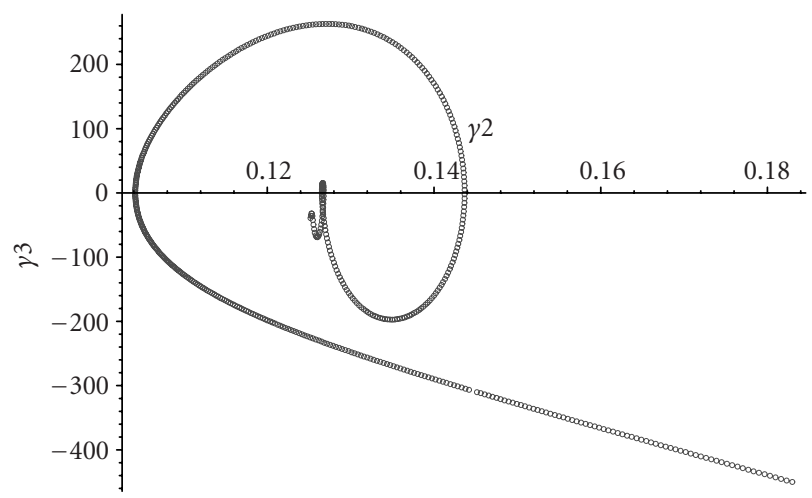

(a)

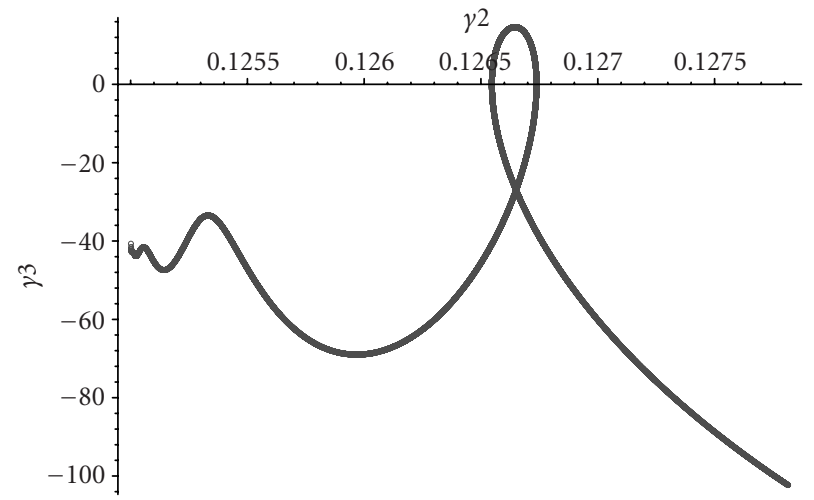

(b)

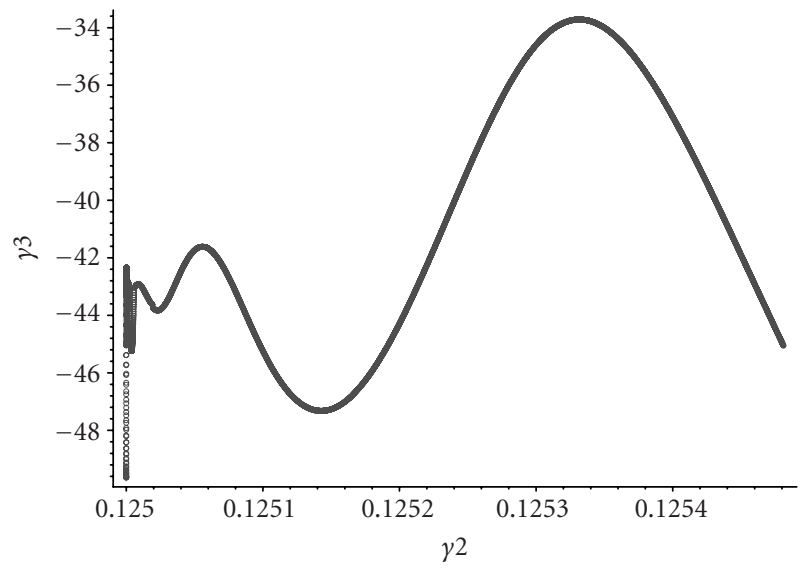

(c)

Figure 5.5. The smaller pattern approached in Figure 5.4 is shown in detail, now displaying a somewhat different general pattern. Here, $\ddot{\varepsilon}$ enters oscillations as $\varepsilon$ approaches the neighborhood of unity. (a) and (b) show enlargements of the inner loop, and (c) shows the endpoint in more detail. 


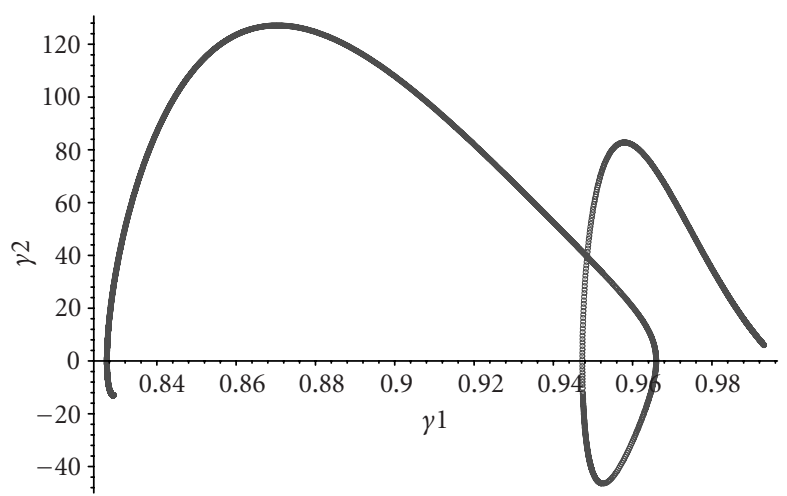

Figure 5.6. $\dot{\varepsilon}, \varepsilon$ plane in the $\tau$ interval represented in Figure 5.2.

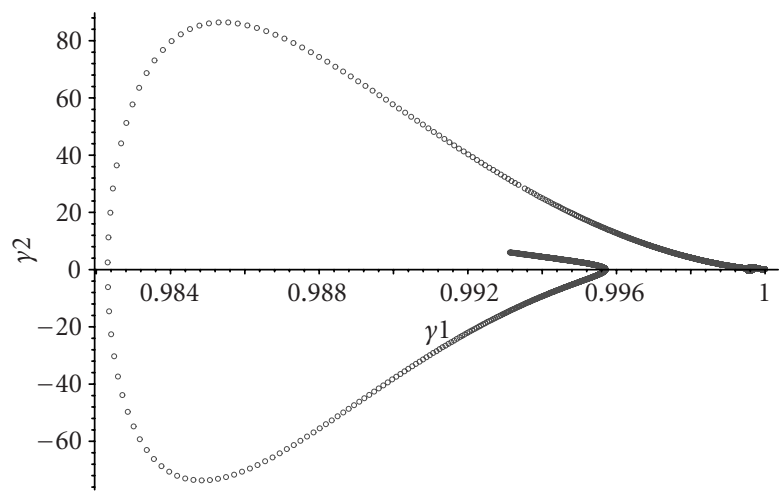

(a)

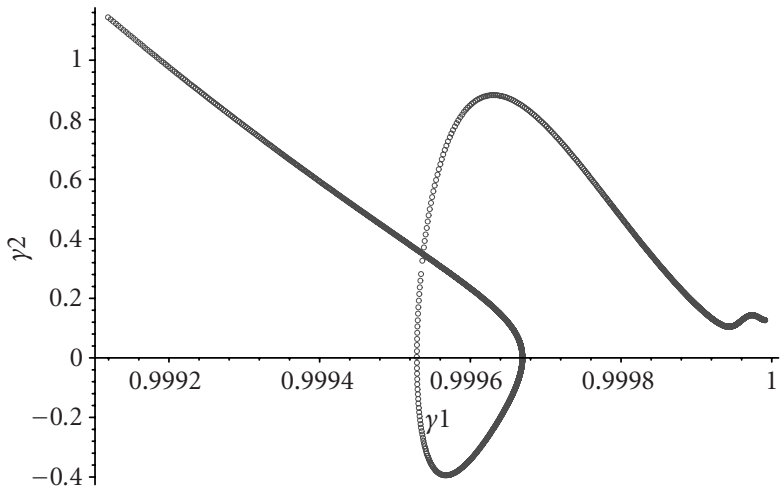

(b)

Figure 5.7. (a) $\dot{\varepsilon}, \varepsilon$ plane in the $\tau$ interval represented in Figure 5.3; (b) $\dot{\varepsilon}, \varepsilon$ plane in the $\tau$ interval represented in Figure 5.4, including the details corresponding to Figure 5.5. 


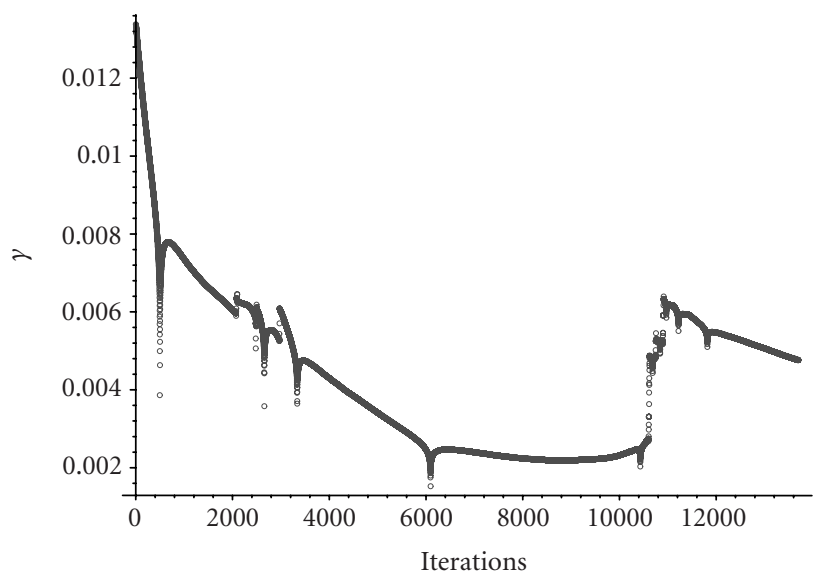

Figure 5.8. The largest averaged Lyapunov exponent (the values must be divided by the size of the interval $10^{-7}$ ) as a function of the accumulated $\tau$. We have used interval $0.4 \times 10^{-6}$. Note the sharp jump at approximately 10,400 steps, indicating the development of a very large instability in this neighborhood (the additional terms here are divided by $10^{4}$, the number of time steps).

We have computed the global Lyapunov exponents according to the standard procedures; recognizing, however, that there appear to be (at least) two levels of behavior types, we have studied the integration on phase space for $\varepsilon$ somewhat less than unity since the integration diverges for $\varepsilon$ close to one. For $\varepsilon$ very close to one, in fact, due to the relation $d s=(1-\varepsilon) d \tau$, this neighborhood contributes very little to the development of the observed orbits.

In this calculation, we have computed the largest Lyapunov exponent (positive) by studying the average separation of the orbits associated with nearby initial conditions [26]. We show the development of the Lyapunov exponent as the average is taken over an increasing interval in Figure 5.8 as data is accumulated up to 13800 iterations (at least two cycles on the attractor). Note that there are intervals of relative stability and very strong instability.

Starting the calculation with different initial conditions, we found very similar dynamical behavior, confirming the existence of the apparent attractor.

We have also studied the time series associated with the three variables of the phase space, shown in Figures 5.9, 5.10, and 5.11. The autocorrelation functions for $\ddot{\varepsilon}$ and $\dot{\varepsilon}$ fall off quickly giving characteristic correlation times; the autocorrelation function for $\varepsilon$ does not fall off faster than linearly, but the time series shows significant structure resembling the phase space structure (subtracting out the local average from the function $\varepsilon$ generates a deviation function that does indeed show a falloff of the autocorrelation function). We have used these scales, for 20 time steps (for $\ddot{\varepsilon}$ and $\varepsilon$ ) and 40 time steps (for $\dot{\varepsilon}$ ), to construct a three-dimensional delay phase space for the time series for each of these functions.

In Figures 5.12(a) and 5.12(b), we show the behavior of solution of the autonomous equation for $\dot{\varepsilon}$ versus $\varepsilon$ and $\ddot{\varepsilon}$ versus $\dot{\varepsilon}$. These results are very similar in form to those given above, demonstrating stability of the results. 
L. P. Horwitz et al. 201

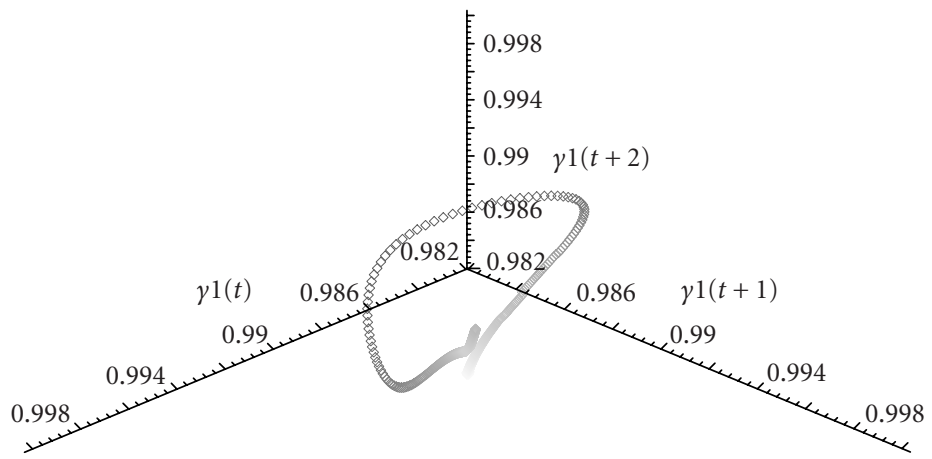

Figure 5.9. The time series for $\varepsilon(\tau)$ on a three-dimensional space corresponding to shift by 20 time steps.

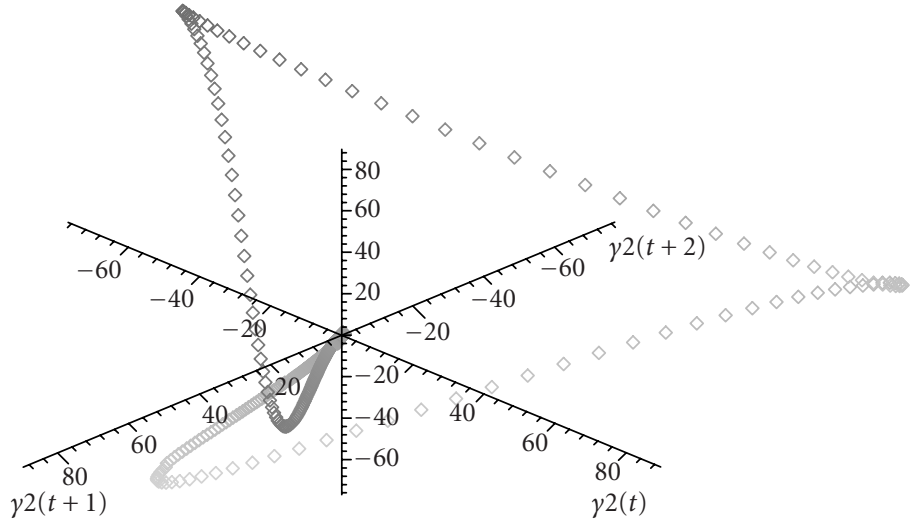

Figure 5.10. The time series for $\dot{\varepsilon}(\tau)$ on a three-dimensional space corresponding to shift by 40 time steps.

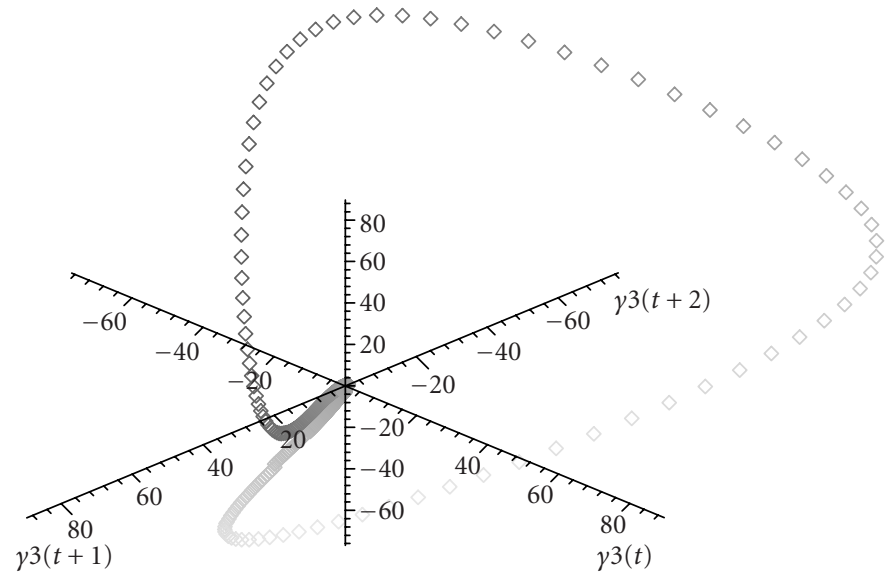

Figure 5.11. The time series for $\ddot{\varepsilon}(\tau)$ on a three-dimensional space corresponding to shift by 20 time steps. 


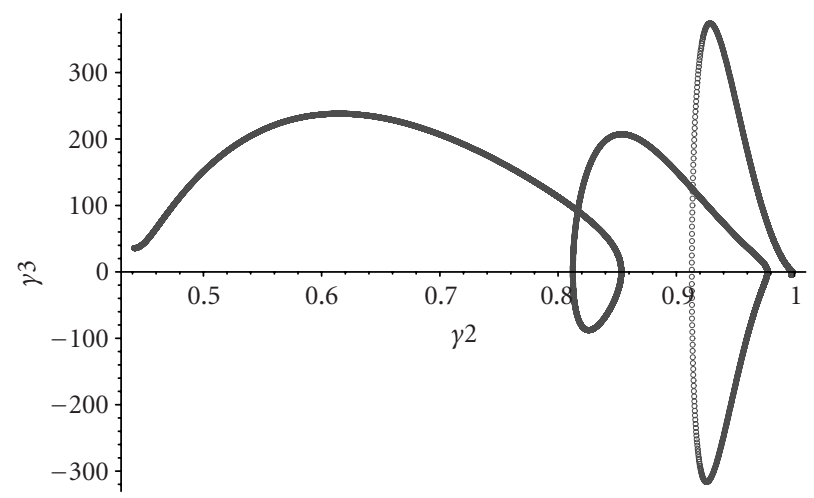

(a)

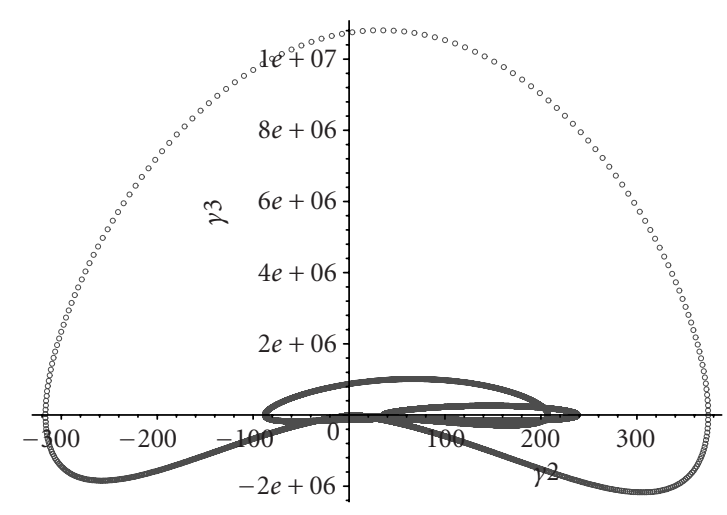

(b)

Figure 5.12. Showing $\dot{\varepsilon}$ versus $\varepsilon$ and $\ddot{\varepsilon}$ versus $\dot{\varepsilon}$, we have changed the initial conditions and found a similar pattern.

\section{Discussion}

We have examined the equations of motion generated for the classical relativistic charged particle, taking into account dynamically generated variations in the mass of the particle, and the existence of a fifth electromagnetic-type potential essentially reflecting the gauge degree of freedom of the variable mass. We have focussed our attention on the autonomous equation for the off-shell mass deviation defined in (2.17) and (2.18) as an indication of the dynamical behavior of the system, and shown that there indeed appears to be a highly complex strange attractor. It is our conjecture that the formation of dynamical attractors of this type in the orbit equations as well will lead to a macroscopically smooth behavior of the perturbed relativistic charged particle, possibly associated with an effective nonzero size, as discussed, for example, by Rohrlich [20, 21, 22]. Some evidence for such a smooth behavior appears in the very large effective mass generated near 
$\varepsilon \cong 1$, stabilizing the effect of the Lorentz forces, as well as our preliminary result that the larger accelerations emerging in derivatives with respect to $\tau$ are strongly damped, in this neighborhood of $\varepsilon$, in the transformation to motion seen as velocity $(d \mathbf{x} / d t)$ in a particular frame.

Our investigation of the phase space of the autonomous equation used somewhat arbitrary initial conditions, and we find that changing these conditions somewhat does not affect the general pattern of the results. Moreover, since $\ddot{\varepsilon}$ depends on the third derivative of $x^{\mu}(\tau)$, this initial condition should not be taken as arbitrary, but as determined by the results for the orbit (initial conditions for the orbit depend only on $x^{\mu}$ and its first two derivatives). Computing the orbit for a typical choice of initial conditions, and using the result to fix $\ddot{\varepsilon}$, we find that the corresponding solution of the autonomous equation indeed lies in the attractor. The results of our investigation of the autonomous equation should therefore be valid for initial conditions that obey the physical constraints imposed by the equations of motion as well.

We have specified all of the dynamical equations here, but a complete investigation must await further planned work with more powerful computing facilities and procedures.

\section{Acknowledgments}

L. P. Horwitz would like to thank the Institute for Advanced Study, Princeton, NJ, for partial support, and Steve Adler for his hospitality, during his visit in the spring semester (2003) when much of this work was done. We would also like to thank Otto Rössler and René Thoma for helpful communications.

\section{References}

[1] M. Abraham, Theorie der Elektrizität. II. Elektromagnetische Theorie der Strahlung, B. G. Teubner, Leipzig, 1905.

[2] J. M. Aguirregabiria, Solving forward Lorentz-Dirac-like equations, J. Phys. A 30 (1997), no. 7, 2391-2402.

[3] C. Beck, Spatio-Temporal Chaos and Vacuum Fluctuations of Quantized Fields, Advanced Series in Nonlinear Dynamics, vol. 21, World Scientific, Singapore, 2002.

[4] L. Burakovsky, L. P. Horwitz, and W. C. Schieve, New relativistic high-temperature Bose-Einstein condensation, Phys. Rev. D 54 (1996), no. 6, 4029-4038.

[5] P. A. M. Dirac, Classical theory of radiating electrons, Proc. Roy. Soc. London Ser. A 167 (1938), 148-169.

[6] R. P. Feynman, Space-time approach to non-relativistic quantum mechanics, Rev. Modern Physics 20 (1948), 367-387.

[7] A. Gupta and T. Padmanabhan, Radiation from a charged particle and radiation reaction reexamined, Phys. Rev. D 57 (1998), no. 12, 7241-7250.

[8] L. P. Horwitz, The unstable system in relativistic quantum mechanics, Found. Phys. 25 (1995), no. 1,39-65.

[9] J. D. Jackson, Classical Electrodynamics, 2nd ed., John Wiley \& Sons, New York, 1975.

[10] M. C. Land, N. Shnerb, and L. P. Horwitz, On Feynman's approach to the foundations of gauge theory, J. Math. Phys. 36 (1995), no. 7, 3263-3288. 
[11] H. Levine, E. J. Moniz, and D. H. Sharp, Motion of extended charges in classical electrodynamics, Amer. J. Phys. 45 (1977), 75-78.

[12] E. Lorenz, Deterministic nonperiodic flow, J. Atmospheric Sci. 20 (1963), no. 2, 130-148.

[13] B. Mashhoon, The hypothesis of locality in relativistic physics, Phys. Lett. A 145 (1990), no. 4, 147-153.

[14] _ Nonlocal theory of accelerated observers, Phys. Rev. A 47 (1993), no. 5, 4498-4501.

[15] , Proc. VII Brazilian School of Cosmology and Gravitation, Editions Frontières, Paris, 1994.

[16] C. W. Misner, K. S. Thorne, and J. A. Wheeler, Gravitation, W. H. Freeman, California, 1973.

[17] O. Oron and L. P. Horwitz, Classical radiation reaction off-shell corrections to the covariant Lorentz force, Phys. Lett. A 280 (2001), no. 5-6, 265-270.

[18] Eikonal approximation to $5 D$ wave equations and the $4 D$ space-time metric, Found. Phys. 33 (2003), 1323-1338.

[19] F. Rohrlich, Classical Charged Particles, Addison-Wesley Publishing, Massachusetts, 1965.

[20] - The unreasonable effectiveness of physical intuition: success while ignoring objections, Found. Phys. 26 (1996), no. 12, 1617-1626.

[21] Classical self-force, Phys. Rev. D (3) 60 (1999), no. 8, 084017, 5 pp.

[22] - The self-force and radiation reaction, Amer. J. Phys. 68 (2000), no. 12, 1109-1112.

[23] D. Saad, L. P. Horwitz, and R. I. Arshansky, Off-shell electromagnetism in manifestly covariant relativistic quantum mechanics, Found. Phys. 19 (1989), no. 10, 1125-1149.

[24] N. Shnerb and L. P. Horwitz, Canonical quantization of four-and five-dimensional U(1) gauge theories, Phys. Rev. A (3) 48 (1993), no. 6, 4068-4074.

[25] A. A. Sokolov and I. M. Ternov, Radiation from Relativistic Electrons, American Institute of Physics Translation Series, American Institute of Physics, New York, 1986.

[26] J. C. Sprott, Chaos and Time-Series Analysis, Oxford University Press, New York, 2003.

[27] E. C. G. Stueckelberg, La signification du temps propre en mécanique: ondulatoire, Helv. Phys. Acta 14 (1941), 321-322 (French).

[28] - Remarque à propos de la création de paires de particules en théorie de relativité, Helv. Phys. Acta 14 (1941), 588-594 (French).

[29] D. Villarroel, Solutions without preacceleration to the one-dimensional Lorentz-Dirac equation, Phys. Rev. A (3) 55 (1997), no. 5, 3333-3340.

[30] A. D. Yaghjian, Relativistic Dynamics of a Charged Sphere, Springer-Verlag, Berlin, 1992.

L. P. Horwitz: School of Physics and Astronomy, Raymond and Beverly Sackler Faculty of Exact Sciences, Tel Aviv University, Ramat Aviv 69978, Israel

E-mail address: larry@post.tau.ac.il

N. Katz: Department of Physics, Bar-Ilan University, Ramat Gan 52900, Israel

E-mail address: natikatz23@hotmail.co.il

O. Oron: School of Physics and Astronomy, Raymond and Beverly Sackler Faculty of Exact Sciences, Tel Aviv University, Ramat Aviv 69978, Israel

E-mail address: orior@post.tau.ac.il 


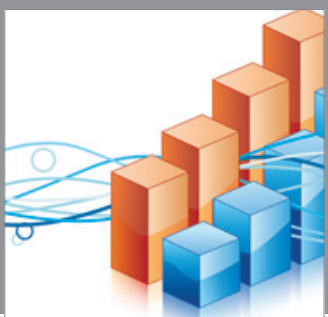

Advances in

Operations Research

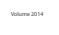

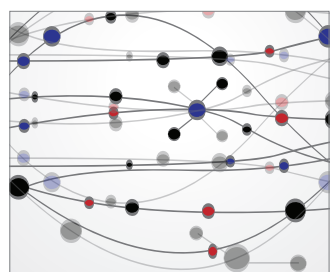

\section{The Scientific} World Journal
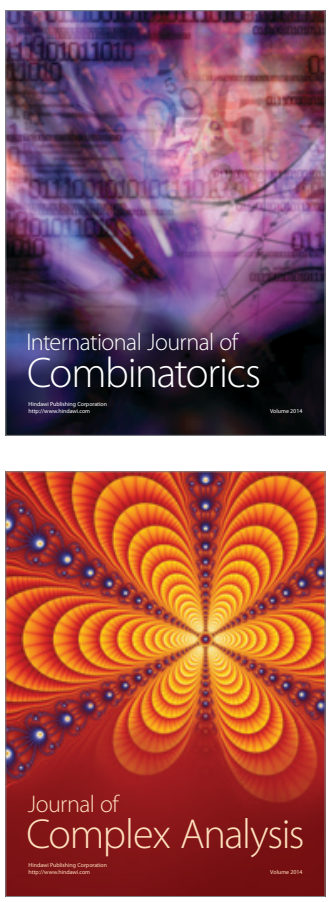

International Journal of

Mathematics and

Mathematical

Sciences
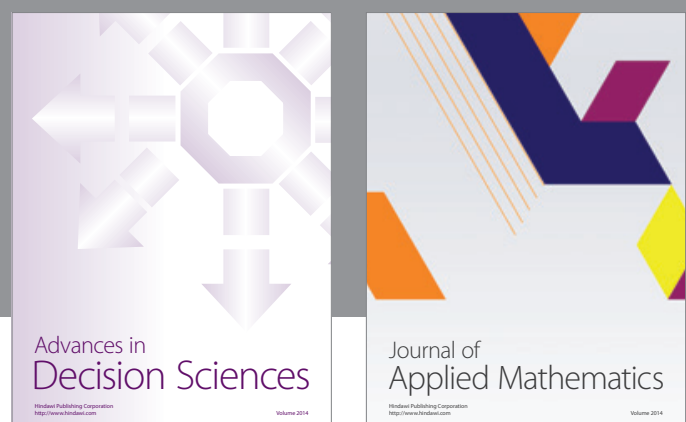

Journal of

Applied Mathematics
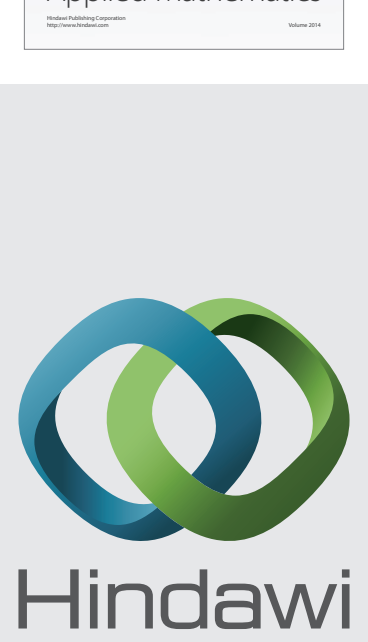

Submit your manuscripts at http://www.hindawi.com
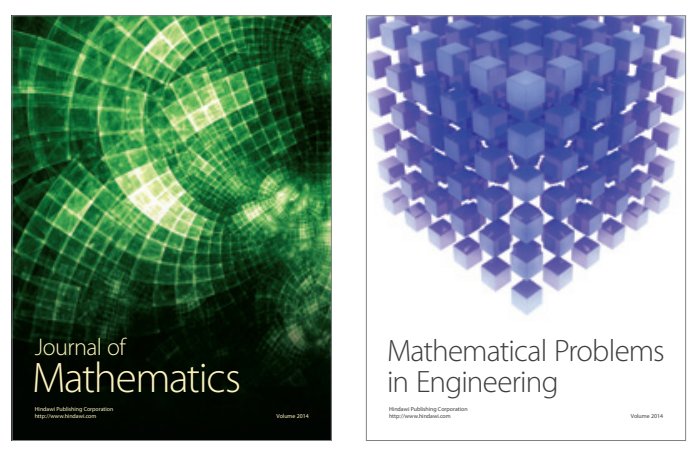

Mathematical Problems in Engineering
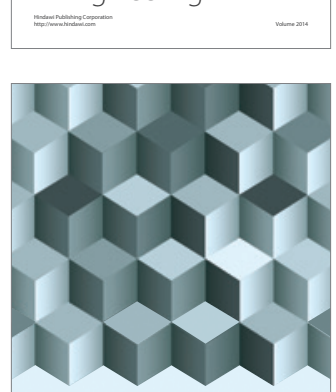

Journal of

Function Spaces
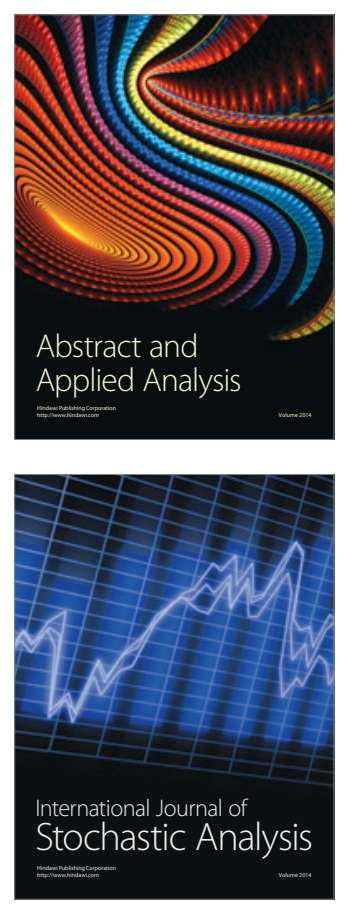

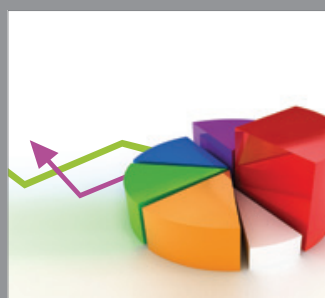

ournal of

Probability and Statistics

Promensencen
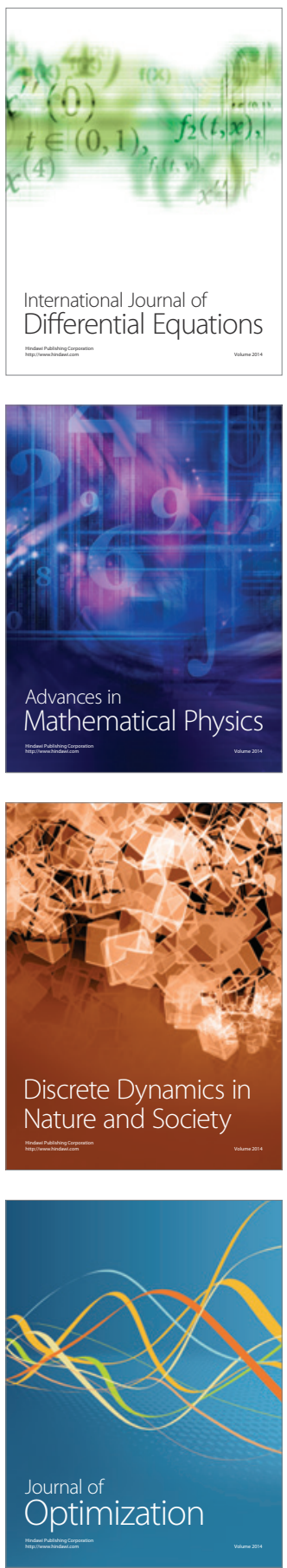